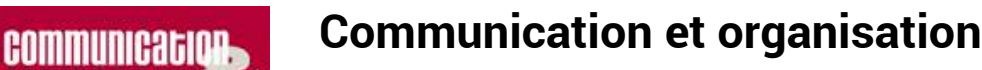

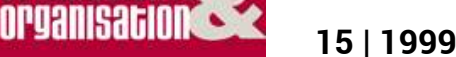

Qualité et communication

\title{
Qualité et communication
}

Jean-Pierre Callégari

\section{OpenEdition}

Journals

Édition électronique

URL : http://journals.openedition.org/communicationorganisation/2173

DOI : 10.4000/communicationorganisation.2173

ISSN : 1775-3546

\section{Éditeur}

Presses universitaires de Bordeaux

\section{Édition imprimée}

Date de publication : 1 mai 1999

ISSN : 1168-5549

\section{Référence électronique}

Jean-Pierre Callégari, "Qualité et communication », Communication et organisation [En ligne], 15 | 1999, mis en ligne le 26 mars 2012, consulté le 19 avril 2019. URL : http://journals.openedition.org/ communicationorganisation/2173 ; DOI : 10.4000/communicationorganisation.2173

Ce document a été généré automatiquement le 19 avril 2019

(c) Presses universitaires de Bordeaux 


\title{
Qualité et communication
}

\author{
Jean-Pierre Callégari
}

1 Dans les entreprises encore fortement imprégnées par le taylorisme, on résume souvent la qualité à un ensemble de procédures formalisées en groupes de travail, rassemblées dans un manuel, applicables car élaborées dans le respect des normes nationales et internationales, mises à jour et vérifiées. Ainsi la qualité est devenue une « démarche à manager ", un moyen de positionnement sur le marché, un enjeu économique et social, pendant que la communication, marquée par la théorie du projectile selon l'expression de G. Willet, est souvent perçue comme une modalité de fonctionnement.

Dans le même temps, Qualité et Communication sont presque devenues des mythes dont les logiques se sont croisées et ont retenti interactivement sur les modes d'organisation jusqu'au management de projet. En fait, l'histoire n'a pas attendu les normes; avant la qualité du produit, il y avait déjà le souci du produit de qualité. Elle ne s'est pas arrêtée. La communication homme-machine a fait prendre conscience que l'homme-utilisateur pouvait devenir acteur et ses besoins une finalité à concilier avec d'autres impératifs, d'où un discours à construire pour intégrer et adapter des contenus à diffuser.

3 Le GREC/O remercie vivement les auteurs d'articles et témoignages. Ils se sont faits l'écho de cette évolution et l'analysent finement. Jean-Pierre Boutinet ouvre ce dossier qualité et communication en croisant ces deux concepts avec celui de projet pour expliciter et rendre manifeste un fond latent. Hélène Giroux et James R. Taylor enrichissent le débat en s'appuyant sur la traduction, application plus habituellement réservée à la sociologie des sciences exactes et de gestion comme le rappelle Yves Winkin. En lisant leur article on perçoit cependant comme un écho de l'ingénierie sociale du travail. Patrick Laurens explique comment et selon quels principes, il faut promouvoir un service de formation qui prépare des acteurs de qualité compétents et satisfaits. Marie-Reine Boudarel rappelle que ceux-ci peuvent aussi apprendre sur le terrain, au quotidien, en commençant par une méthode qualité plus adaptée, intéressante dans sa simplicité. Les choses se complexifiant, Pierre Michel Riccio veut éclairer le chemin en proposant une méthodologie à l'interface de la communication et des technologies de l'information. Jean-Marc Laubin, Alain Durand, Sylvie Leleu-Merviel approfondissent et ouvrent toute grande la porte sur les Nouvelles Technologies de l'Information et de la Communication, 
pour proposer un mode d'évaluation à même de renseigner sur la justesse et la qualité de ce que l'on fait. Plus succinctement, Isabelle Orgogozo invite au changement des mentalités et recommande la communication-écoute pour comprendre.

4 En ouvrant son quinzième dossier sur Qualité et Communication, la Revue du GREC/O n'ambitionnait pas explicitement l'exhaustivité, mais le champ se trouve assez largement couvert et riche de promesses. La revue invitait à s'interroger sur les deux concepts et leurs rapports régénérant, plus précisément sur la nature, le rôle et la place de communication. Le chemin paraît bien tracé.

5 Loin de mésestimer l'importance de la communication-modalité de fonctionnement dans la réalisation de la qualité, de l'adéquation à ménager entre qualité voulue et accomplie d'une part, qualité attendue et perçue d'autre part, nous souhaitions que les auteurs se laissent davantage interpeller par la norme ISO 8402 révisée, définissant la qualité comme l'ensemble « des caractéristiques d'une entité qui lui confèrent l'aptitude à satisfaire des besoins exprimés et implicites ». Certes la démarche reste une condition nécessaire, mais au-delà du processus, le terme d'entité suggère un peu plus l'homme, surtout la nature sinon l'essence du concept.

6 Depuis quelques années, il nous semble en effet que la qualité représente un sujet pour la communication, également facteur d'évolution culturelle dans la démarche qualité d'une entreprise. Si les deux concepts interfèrent, l'un et l'autre paraissent relever d'un état d'esprit, d'une manière d'être, d'une culture qu'ils contribuent interactivement à fonder. Ils sont fondamentalement au cœur d'une démarche qualité qui ne saurait se résumer à une partition. Les auteurs du dossier avaient perçu cela et s'inscrivent dans la problématique.

7 - Spécialiste du management par projet, Jean Pierre Boultinait dresse avec conviction et compétence, un historique qui resitue assez bien les concepts de projet, qualité, communication, comme participant interactivement d'une même culture. Il commence naturellement par souligner l'ambiguïté du projet qui croise tour à tour des logiques innovantes et coercitives, émancipatrices et assujettissantes. Il explicite ensuite plus longuement le concept de qualité dans sa double dimension d'attributs particularisants mais relatifs, de spécificités essentielles qui singularisent. L'articulation entre ces deux concepts conduit à la communication, troisième «socle» en fait transversal aux précédents, révélateur, d'une lutte contre l'équivoque pour imposer la transparence, et d'un souci de dépasser les exigences nécessaires pour laisser un espace à l'acteur, autrement dit à l'homme qui projette et anime.

- L'article d'Hélène Giroux et James R. Taylor sur l'évolution du discours qualité à partir des travaux de Latour sur la traduction, intéresse par la clarté et l'originalité de l'approche. De surcroît, il propose une analyse historique et circonstanciée du concept qualité de 1977 à 1992 et au delà, devient implicitement une analyse de la communication avec une thèse sur l'incidence de la traduction dans la présentation du contenu. Au travers de la façon dont la qualité est présentée et rendue perceptible, les auteurs montrent comment on intéresse ses lecteurs, comment on rend un discours crédible et mobilisateur, en fait comment on crée une interrelation énonciateur-énonciataire avec un contenu inévitablement transdisciplinaire qui, par sa forme, épouse les courants d'un moment sans rien renier du fond et des objectifs fondamentaux.

9 - Patrick Laurens part d'une logique de conformité et de nécessaire maîtrise des processus, dresse un état de l'art de la démarche qualité reposant sur une communication 
interne d'entreprise, instrument rationalisé au service d'un idéal relationnel, construit entre désir et demande par l'objectivation d'un compromis qui rencontre la « rationalité limitée » et amène les acteurs à coproduire l'information qu'ils appréhendent. Ce faisant, il passe aisément à la qualité de la formation, bien immatériel. La qualité du service de formation ne peut pas plus être laissée au hasard que la qualité voulue et accomplie par l'entreprise. Toutefois, en recommandant un projet communicationnel dans lequel les acteurs, clients, apprenants, coopéreraient pour construire une réalité qui n'est pas entièrement "déjà-là ", il introduit implicitement le concept de service associé dans la qualité du service : il implique judicieusement la distinction entre la discussion sur le type et les modalités de la formation et celle-ci, soit le produit lui-même, souvent rapidement désigné comme le service.

- Marie Reine Boudarel précise le rôle de la communication au travers d'expérimentations menées en petites et moyennes entreprises, de la méthode japonaise dite des $5 \mathrm{~S}$ - en français: débarrasser, ranger, nettoyer, se nettoyer personnellement, systématiser. Véritable témoignage, l'article explique comment l'introduction d'une méthode qualité dans les préliminaires d'une démarche concrète plus globale, permet de faire l'apprentissage, au quotidien, de la communication dans certaines dimensions essentielles: facteur d'évolution et dépassement des freins, mise en relation, responsabilisation individuelle et collective, maturation de l'identité par l'amélioration et de l'affirmation de soi.

11 - Informaticien d'origine, Pierre Michel Riccio propose une méthode «empiricoinductive » d'élaboration d'un système qualité, qui jette un pont entre les sept processus définis par Alex Mucchielli pour la communication, et le langage U.M.L. (Unified Modelling Language). Après un état de l'art nécessaire, l'auteur souligne précisément les améliorations, complémentarités, nouveautés et interactions, qui fondent l'intérêt d'un croisement dont résulte une méthode systémique, validée en entreprise, sans doute culturellement conciliatrice pour nombre de praticiens et chercheurs en Sciences de l'Information et de la Communication.

12 -Jean Marc Laubin, Alain Durand et Sylvie Leleu-Merviel, développent une méthodologie précise, révélatrice d'une démarche qualité bien balisée, dérivée de l'analyse fonctionnelle, illustrée par de nombreux exemples issus du multimédia mais transposables à d'autres domaines. Leur article est encore exemplaire quand il explicite un mode d'évaluation à même de rendre une démarche de communication plus efficiente, jusque dans la construction du sens en fonction d'un récepteur.

13 - Dans une analyse qui relève principalement de l'histoire et de la sociologie, Isabelle Orgogozo apporte un témoignage intéressant, souligne l'urgence de changer les mentalités en même temps que les modes d'organisation des entreprises, pour développer une communication fondée sur l'écoute, l'échange et la volonté de comprendre plutôt que transmettre et chercher à convaincre. Les nouvelles technologies de l'information pourraient représenter une opportunité, mais l'apprentissage passe notamment par les cercles de qualité. 


\section{AUTEUR}

\section{JEAN-PIERRE CALLÉGARI}

Université de Valenciennes et du Hainaut Cambrésis 